\title{
A device for continuous monitoring of pupil dilation
}

\author{
H. LOWETH \\ University of Warwick. Warwick, England
}

This report describes the design and functioning of a pupillometer for use in clinical and experimental psychology.

A number of pupillometers have been developed over the last 15 years. Hess (1965), for example, used a photographic record of pupil size. This method is unsuitable for clinical and some experimental uses because the results are not available until the film is processed. Lowenstein and Loewenfeld (1958) used an electronic scanning method; however, this equipment is expensive, and the Ss' head must be rigidly clamped.

This report describes a pupillometer that has some advantages over other designs and is suitable for everyday use.

\section{DESCRIPTION OF PUPILLOMETER}

Slides are projected onto a screen in front of the $\mathrm{S}$ (see Fig. 1). The pupillometer records the pupillary response to these slides. An infrared light positioned behind the screen illuminates the $S$ through a small hole in the center of the screen. The $S$ wears spectacles that are opaque except for an 8 -mm-diam hole in front of each eye. These holes allow an $8-\mathrm{mm}$ circle of infrared light to fall onto each iris. If the pupil dilates, then the light reflected from the iris decreases. This reflected light is measured by two phototransistors (Fairchild FPT 120) positioned on either side of one eye. It is important that the white of the eye (the sclera) not fall within the light spot and also that the pupil not move out of the light spot; so eye movement must be limited. This is achieved by reducing the size of the screen suitably.

Figure 2 shows the circuit diagram. The FPT 120 phototransistors are connected to "buffer" amplifiers (Texas SN 741). These prevent stray pickup in the cable between the spectacles and the bench. The adder then summates the two outputs. One arm of the adder has a potentiometer, the "balance pot," which is used to compensate for any difference in the phototransistor gains. The filter reduces the time necessary for the chart recorder to return to its original position after a blink. A two-track recorder is used: one track records pupil size and the other indicates when each slide is projected. The "light-level" potentiometer is used to adjust the circuit to any light level.

Calibration consists of two steps. (1) The gain of the pupillometer circuit is measured. The spectacles are laid on a surface, the phototransistors are illuminated, and the variations in output that result from variations in light intensity are recorded. (2) The pupillometer is calibrated for each new $S$. The $S$ wears the spectacles, and a plain gray slide is projected onto the screen. The $S$ 's pupil size is measured by an $O$ while the output is recorded; hence, the output for one particular pupil area is known. The output for all other pupil areas can then be calculated using the results from Step 1 .

\section{RESULTS}

Figure 3 shows a typical printout obtained by a $S$ fixating a simple mathematical problem and providing the solution mentally. The pupil dilates as soon as the problem is projected and continues to dilate until the $S$ gives his answer, following which the pupil returns to its original size. The marked downward deflections indicate eye blinks.

The pupillometer was found to produce readings accurate to within $7 \%$ of true pupil size.

\section{APPRAISAL OF PUPILLOMETER}

The above results suggest that the pupillometer is well suited to clinical and experimental applications. Further development work is desirable, so mention is made of

Fig. 1. Plan view of pupillometer.

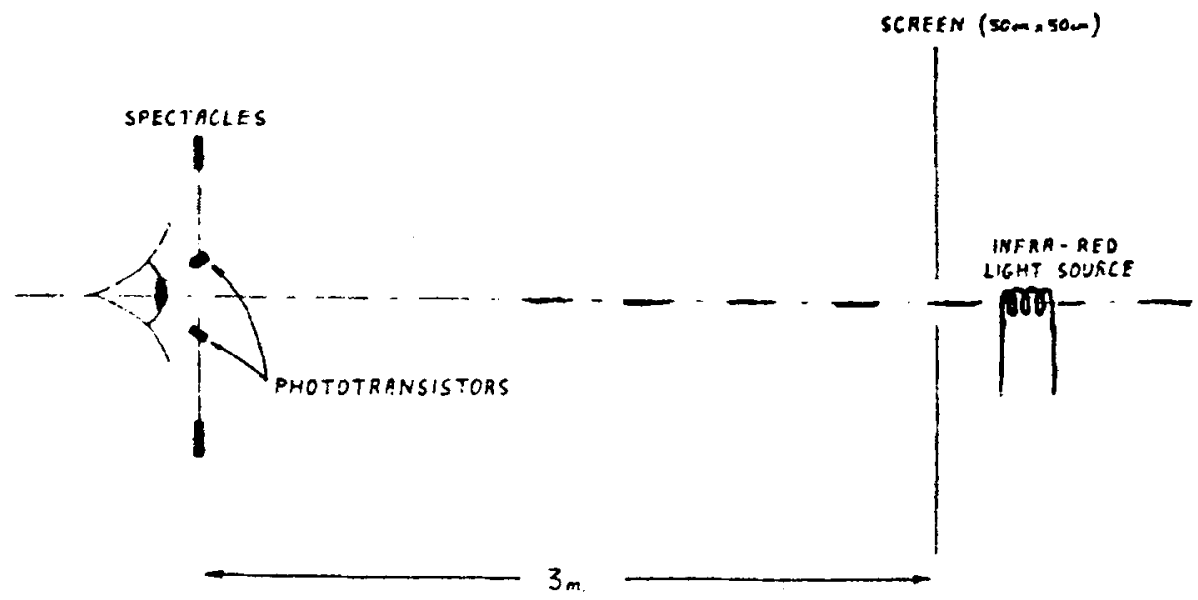




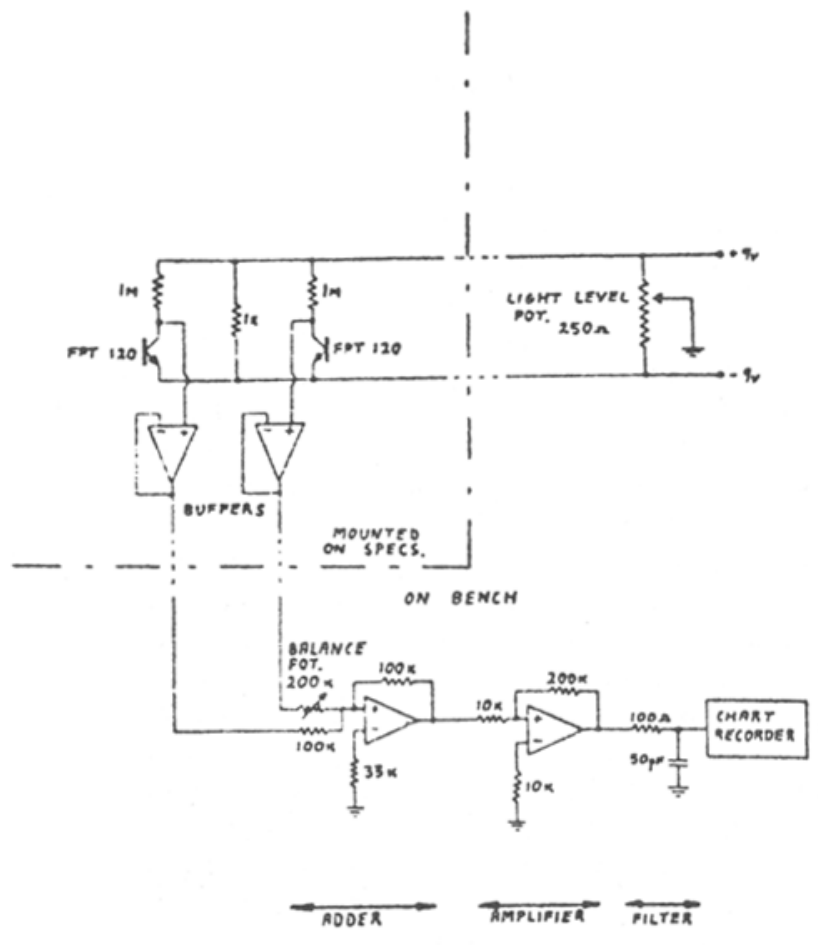

Fig. 2. Circuit diagram of pupillometer.

two problems the investigator must consider: (1) The overall brightness of each slide must be matched to some reference level, so that no pupil variations occur when a new slide is projected. This brightness level must be reduced to an acceptable degree in order to minimize the pupil variations that result from viewing a bright area in a dark slide. (2) Corneal reflection: (a) Light from the screen is reflected by the cornea onto the phototransistors. This is eliminated by placing an infrared filter over each phototransistor. (b) Light from the infrared source is also reflected onto the phototransistors; however, this remains virtually constant, even though the eye moves, and so does not affect the output.

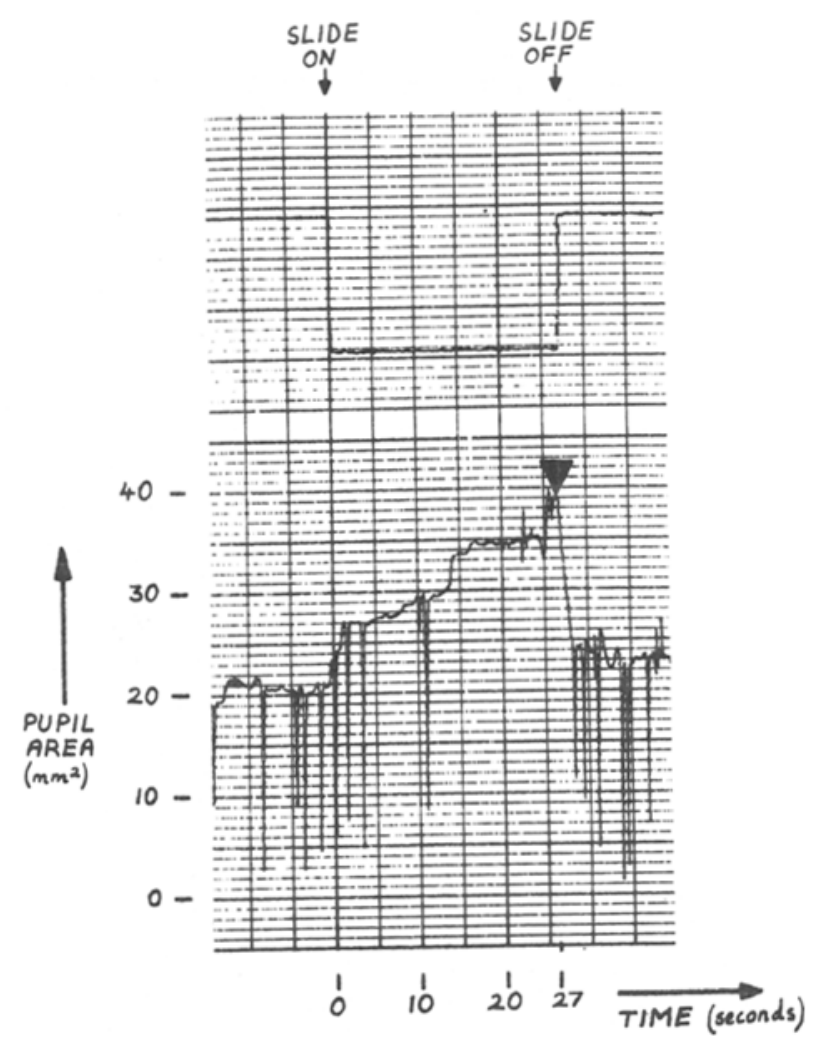

Fig. 3. The pupil size of a $S$ during the solution of $17 \times 17$ (A shows when the $S$ gave the answer).

The pupillometer described in this report has several advantages over other designs of pupillometer: it is simpler, it allows the $S$ freedom of movement, and it provides a continuous record of pupil size.

\section{REFERENCES}

Hess, E. H. Attitude and pupil size. Scientific American, 1965 $212,400-411$.

Lowenstein, O., \& Loewenfeld, I. E. Electronic pupillography. Archives of Ophthalmology, 1958, 59, 352-363.

(Received for publication May 16, 1973; revision received September 17, 1973.) 